\title{
Arbeitgeberverbände - auf dem Weg in den Dualismus?
}

\author{
Arbeitgeberverbände sind aus dem deutschen System der Arbeitsbeziehungen kaum weg- \\ zudenken, denn sie wirken in einem umfassenden Sinne an der Regulierung der Arbeits- \\ welt mit. Dieser Beitrag geht am Beispiel der beiden miteinander verbundenen Prozesse \\ „innere“ und „äußere Erosion“ der Arbeitgeberverbände der Frage nach, ob diese ihre \\ zentrale Rolle auch weiterhin werden wahrnehmen können. Am Beispiel der generellen \\ Mitgliederentwicklung der Verbände, aber auch anhand der Möglichkeiten zur selektiven \\ Abwahl der Tarifbindung (OT-Mitgliedschaften) wird die These diskutiert, dass die Ar- \\ beitgeberverbände als eine Kerninstitution im System der industriellen Beziehungen ei- \\ nen gespaltenen Entwicklungspfad eingeschlagen hätten.
}

MARTIN BEHRENS

\section{Einleitung}

Arbeitgeberverbände haben Gewicht: sei es als Partner der Gewerkschaften in Tarifverhandlungen, als durchsetzungsstarke „pressure group“ bei der Durchsetzung unternehmensfreundlicher Gesetzgebung, oder sei es auch als tief im deutschen Institutionensystem verankerte Kollektivakteure mit Sitz und Stimme in Rundfunkräten, Arbeitsgerichten sowie in den Selbstverwaltungsorganen der Sozialversicherung.

Zwar vertreten Arbeitgeberverbände über ihre Unternehmensmitglieder besonders finanz- und durchsetzungsstarke Interessen, jedoch lassen sich diese Ausgangsinteressen nicht einfach auf dem Wege schlichter Aggregation in eine starke kollektive Interessenvertretung überführen. Wie nicht zuletzt Meldungen über eine rückläufige Mitgliedschaft bei den Arbeitgeberverbänden, aber auch wachsende Schwierigkeiten bei der Formulierung einer einheitlichen, branchenübergreifenden Arbeitgeberpolitik verdeutlichen, sind zunehmend Zweifel angebracht, ob die deutsche Sozialpartnerschaft zukünftig auf starke Arbeitgeberverbände als Schlüsselakteure im System der Verbandstarifverträge wird bauen können. Im Folgenden wird daher die Frage nach Ausmaß, Form und Mustern der beobachteten Erosion von Arbeitgebermacht gestellt. Es wird die These geprüft, dass sich die zu beobachtende Schwächung von Arbeitgeberverbänden keineswegs als gleichför- miger, universeller Prozess vollzieht, sondern in Form von Segmentierung oder Dualisierung der deutschen Arbeitgeberverbandslandschaft.

Die These einer Dualisierung ist keineswegs neu und wurde zuletzt von Palier/Thelen (2010) und Hassel (2011) vertreten. Dabei grenzen sich insbesondere Palier/Thelen (2010, S. 120) deutlich von Positionen ab, die davon ausgehen, die politische Ökonomie Deutschlands wäre Gegenstand eines umfassenden Prozesses der Liberalisierung grundlegender Strukturen und Institutionen geworden. In ihrer Analyse der Entwicklung von Institutionen in den drei Arenen industrielle Beziehungen, Arbeitsmarkt und sozialstaatliche Regulierung stellen sie eine asymmetrische Struktur des Zerfalls fest. Zwar verliere das deutsche (ebenso wie das französische) Modell seine Fähigkeit, einen umfassenden und einheitlichen sozialen Schutzmechanismus für alle Bürgerinnen und Bürger bereitzustellen, jedoch bedeute dies keineswegs einen generellen Zerfall des traditionellen Systems der industriellen Beziehungen. Auch könne von einem Frontalangriff der Arbeitgeber auf die Kerninstitutionen dieses Systems nicht die Rede sein. Allerdings führten zu beobachtende Erosionstendenzen dazu, dass ein Kern innerhalb der deutschen politischen Ökonomie - zumeist Großunternehmen des verarbeitenden Gewerbes zulasten der Peripherie stabilisiert werde. Die treibende Kraft hinter diesem Prozess, so wird bei Palier/Thelen angedeutet, sei bei der Kooperation zwischen Management und Gewerkschaften/Betriebsräten innerhalb der 
Kernsektoren zu suchen (ebd. S. 139). Wie weiterhin Hassel vermutet, kommt es hierbei zu „producer coalitions“, die letztlich Arbeitsbeziehungen dualisieren: Im Kern leben bestehende Mechanismen der Koordinierung fort, während die Peripherie zum Gegenstand der Liberalisierung gemacht werde (Hassel 2011, S. 10). Als treibende Kräfte für eine solche Stabilisierung der Kerne gelten in beiden Ansätzen betriebliche Bündnisse für Arbeit, wie sie beispielsweise auf Basis tariflicher Öffnungsklauseln etabliert wurden (ebd. S. 12ff.; Palier/Thelen 2010, S. 122ff.). Gewerkschaften werden in dieser Lesart des Dualismus quasi zu Kronzeugen der Abkoppelung bestimmter Sektoren der Ökonomie, wenn nicht gar zu Komplizen eines „local egoism“ (ebd. S. 122). Hierbei, so wiederum Hassel, versäumten es die Gewerkschaften, separate Abmachungen auf Betriebsebene zu verhindern, was wiederum zur Folge hätte, dass sich der Schutz von Kernbelegschaften letztlich als komplementäres Pendant zur Instabilität der Ränder erweise (Hassel 2011, S. 14).

Während also in dieser Dualismus-Literatur die treibenden Kräfte der Veränderung auf der Ebene der Betriebe und Unternehmen gesucht werden, soll es im Folgenden darum gehen, den Einfluss der Arbeitgeberverbände näher zu beleuchten. In einem ersten Schritt (Abschnitt 2) wird nach dem Ausmaß von Erosionstendenzen gefragt und untersucht, inwieweit sich Mitgliederverluste der Verbände in Form von zurückgehender Tarifbindung ausdrücken. Im dritten Abschnitt wird am Beispiel der sogenannten Mitgliedschaften ohne Tarifbindung die „innere Erosion“ der Tarifwilligkeit von Arbeitgeberverbänden thematisiert, bevor schließlich (Abschnitt 4) die Frage nach den Konsequenzen zurückgehender kollektiver Arbeitgebermacht aufgeworfen wird.

\section{2. „Äußere Erosion“: Verbandsflucht oder Reproduktionskrise?}

Belastbare Daten über die direkte Mitgliedschaft von Unternehmen in Arbeitgeberverbänden liegen zwar für eine Mehrzahl der insgesamt ca. 700 Verbände nicht vor, jedoch lassen sich näherungsweise Entwicklungstendenzen anhand von Daten über die Verbreitung der Bindung an durch die Verbände abgeschlossene Flächentarifverträge ablesen. Solche Daten werden für Westdeutschland seit 1993 (für Ostdeutschland seit 1996) regelmäßig im Rahmen des IABBetriebspanels durch das Institut für Arbeitsmarkt- und Berufsforschung erhoben und veröffentlicht. Zusammengefasst lässt sich die Entwicklungstendenz wie folgt charakterisieren: Zwar gab es in West- wie in Ostdeutschland immer wieder einmal kurze Phasen der Stagnation, jedoch kam es insgesamt zu einem fortlaufenden Abschmelzen der Tarifdeckungsraten. Wurden 1996 noch 70 \% der Beschäftigten der westdeutschen Gesamtwirtschaft von einem Verbandstarifvertrag erfasst, sank dieser Anteil bis zum Jahr
2011 auf $54 \%$. Die entsprechenden Anteile für Ostdeutschland betrugen $56 \%$ im Jahr 1996 und 37 \% im Jahr 2011 (Ellguth/Kohaut 2012, S. 300). Ein deutliches Indiz für mögliche Segmentierungstendenzen ergibt sich aus der Betrachtung von Tarifdeckungsraten auf der Ebene einzelner Branchen: Liegt hier der Anteil der Beschäftigten, deren Vergütungen und Arbeitsbedingungen durch einen Verbandstarifvertrag geregelt werden, in den Branchenbereichen Energie/Wasser, Abfall und Bergbau, Finanz- und Versicherungsdienstleistungen sowie Öffentliche Verwaltung/Sozialversicherung noch bei zum Teil deutlich über $70 \%$, so wird mit $16 \%$ der Beschäftigten des Branchenbereichs Information und Kommunikation nur eine kleine Minderheit von Verbandstarifverträgen erfasst (Ellguth/ Kohaut 2012, S. 298). Letztlich unterstützen diese Befunde eine Dualisierungsthese, wonach sich die Verbandslandschaft entlang von Branchenclustern strukturiert.

\subsection{Bestimmungsfaktoren der Verbands- mitgliedschaft}

Analysen zu den Bestimmungsfaktoren der Verbandsmitgliedschaft (oder korrespondierend der Verbandsabstinenz) haben zunächst einmal auf die sich verändernden Dynamiken der Entwicklung der Verbandsmitgliedschaft hingewiesen. So wurde noch kurz nach der deutschen Vereinigung von „Verbandsflucht“ gesprochen, einem Prozess, in dessen Folge eine große Anzahl von Unternehmen ihre Mitgliedschaft in Arbeitgeberverbänden aufgab (Schroeder/Ruppert 1996). So verlor etwa Gesamtmetall, als die wohl bedeutendste deutsche Arbeitgeberorganisation, allein in den fünf Jahren von 1991 bis 199623 \% seiner Mitgliedsunternehmen.

Wie Studien aus den frühen 1990er Jahren zeigen, begründeten Unternehmen die Beendigung ihrer Mitgliedschaft in den Mitgliedsverbänden von Gesamtmetall häufig mit ihrer Unzufriedenheit mit den Verbandstarifverträgen, insbesondere auch mit deren mangelnder Flexibilität gegenüber betrieblichen Erfordernissen (ebd. S. 56). Konflikte innerhalb der Arbeitgeberorganisation entzündeten sich häufig im Bereich der Arbeitszeitregelungen und drückten sich aus in Form von Spannungen zwischen Repräsentanten kleiner und mittelständischer Unternehmen und den Vertretern der Großunternehmen (Thelen 2000).

Lag also der Fokus von Studien der frühen 1990er Jahre noch auf der Beschreibung und Erklärung von Verbandsflucht als bewusstem Arbeitgeberhandeln, so wurden zurückgehende Tarifdeckungsraten später eher im Kontext struktureller Entwicklungen der Unternehmenslandschaft interpretiert. Demnach muss man sich die zurückgehende Verbandsmitgliedschaft nicht als einen Prozess vorstellen, bei dem Verbandsmitglieder ihrer Organisation den Rücken zukehren, sondern als eine fortlaufende und inkrementelle Entwicklung, die sehr stark durch die Unternehmensmerkmale Alter und Größe beeinflusst wird. Länger existierende Unternehmen weisen eine höhere Wahrscheinlichkeit auf, 
einem Arbeitgeberverband anzugehören, als jüngere. Stellen nun Altunternehmen ihre Geschäftstätigkeit ein und scheiden somit aus dem Verband aus, so fällt es schwer, diese Mitglieder durch neue aus den Reihen der neu gegründeten Unternehmen (bzw. der bereits verbandsresistenten Altunternehmen) zu ersetzen (Ellguth/Kohaut 2010). Die daraus resultierende Entwicklung lässt sich eher als "Austrocknen“ oder „Erosion“ denn als „Verbandsflucht“ beschreiben.

Hierbei ist allerdings das Alter der Unternehmen nicht das einzige relevante Merkmal. So hat eine wachsende Zahl von Studien belegt, dass eine überschaubare Anzahl von Schlüsselvariablen positiv mit der Mitgliedschaft eines Unternehmens im Arbeitgeberverband assoziiert werden kann (Schnabel/Wagner 1996; Lehmann 2002; Ellguth/Kohaut 2010). Neben dem Alter des Unternehmens sind die Variablen Unternehmensgröße sowie die Zugehörigkeit zu einem größeren Unternehmensnetzwerk von großer Bedeutung. Es mag überraschen, dass sich kein signifikanter Zusammenhang feststellen lässt zwischen dem wirtschaftlichen Erfolg eines Unternehmens und der Wahrscheinlichkeit seines Austritts aus dem Arbeitgeberverband (Lehmann 2002, S. 171; Ellguth/Kohaut 2010) - anders als dies die Verbandsflucht-Debatte teilweise nahelegt. Würde sich, wie von Teilen der Dualisierungs-Literatur behauptet, hier ein ökonomisch potenter Kern zulasten einer ökonomisch schwächeren Peripherie stabilisieren, wären andere Befunde zu erwarten gewesen, nämlich, dass wirtschaftlich schwache Unternehmen den Verband verlassen, weil sie sich den Tarif einfach nicht mehr leisten können.

Während die bislang dargestellten Forschungsarbeiten auf Strukturmerkmale der einzelnen Unternehmen abzielen, analysiert ein zweiter Forschungsstrang Arbeitgeberverbände selbst als autonome Sphäre des Arbeitgeberhandelns. So kommen Behrens/Helfen (2012) in ihrer Untersuchung der Orientierungen von Arbeitgeberverbandsgeschäftsführungen zu dem Ergebnis, dass diese eine größere Wahrscheinlichkeit aufweisen, dem Konzept der Sozialpartnerschaft positiv gegenüberzustehen, wenn die Verbände einen überdurchschnittlich hohen Organisationsgrad haben, über ausreichende finanzielle/personelle Ressourcen verfügen, eine möglichst breite Palette von Mitgliederinteressen vertreten und schon in der Vergangenheit gute Erfahrungen in der tarifpolitischen Auseinandersetzung mit Gewerkschaften sammeln konnten. Die organisatorische Stärke der Arbeitgeberverbände wiederum - so konnte in einer statistischen Analyse belegt werden - steht stark mit der Frage in Verbindung, wie Arbeitgeberverbände das Feld strukturieren, innerhalb dessen sie Mitgliederinteressen zu vertreten wünschen: So erweist sich eine eher heterogene Zusammensetzung der Mitgliedschaft - das heißt eine Mischung von Klein- und Großunternehmen - als positiv für den Arbeitgeberorganisationsgrad, die Vertretung einer großen Vielzahl unterschiedlicher Branchen innerhalb einer einheitlichen Organisation hingegen als negativ (Behrens/Helfen 2009). In Bezug auf die eingangs präsentierte Dualisierungs- these bedeuteten diese Befunde einen völlig anderen $\mathrm{Zu}$ schnitt von Zentrum und Peripherie als von der Ausgangsthese unterstellt. Der stabile Kern zeichnet sich hier nicht primär durch die besondere Wettbewerbsfähigkeit der Großunternehmen des verarbeitenden Gewerbes aus, sondern - zumindest auf der Ebene der Verbände - durch eine Mischung von Groß- und Kleinunternehmen innerhalb einer einheitlichen Verbandsstruktur.

\subsection{Organisationsleistung der Arbeitgeber- verbände und „Erstreckung“}

Wie bedeutsam aber eine Mitgliedschaft im Arbeitgeberverband für die deutschen Arbeitsbeziehungen ist, lässt sich allein schon an den folgenden Schlüsselmerkmalen ablesen: Gehörten im Jahre 2010 lediglich 18,6 \% der abhängig Beschäftigten einer Gewerkschaft an (gewerkschaftlicher Netto-Organisationsgrad), so profitierten auch noch nach jahrelangem Rückgang der Tarifbindung immerhin noch 62 \% der Beschäftigten von den Produkten der gewerkschaftlichen Tarifpolitik und wurden von einem Tarifvertrag (Verbandstarifvertrag oder Haustarifvertrag) erfasst (Angaben nach ICTWSS-Database 2011). Diese Differenz lässt sich zum kleinen Teil durch die Wirkung von für allgemeinverbindlich erklärten Tarifverträgen begründen (Bispinck 2012), der Löwenanteil geht aber zweifelsohne auf das Konto der hohen Organisationsleistung der Arbeitgeberverbände. Gehört ein Unternehmen erst einmal einem tarifschließenden Arbeitgeberverband an, so erstreckt es für gewöhnlich tarifliche Standards auf die ganze Belegschaft und nicht nur auf jene Beschäftigten, die Mitglied in der tarifschließenden Gewerkschaft sind. Der Effekt dieser arbeitgeberseitigen Erstreckung ist unterschiedlich stark ausgeprägt, wie die folgende Analyse auf Basis der Daten der WSI-Betriebsrätebefragung 2010 zeigt. Die nun folgenden Angaben beziehen sich lediglich auf solche Betriebe, die mehr als 20 Personen beschäftigen und über einen Betriebsrat verfügen. Klein- und betriebsratslose Betriebe (die letztere Kategorie repräsentiert immerhin 59 \% der Beschäftigten) können also in die Analyse nicht mit einbezogen werden. Diese Einschränkung ist sicherlich erheblich, weil die untersuchten Betriebe allein schon wegen der Existenz eines Betriebsrates in ihrer Mehrzahl zu den „Kernen“ gezählt werden dürften, letztlich ist die Verwendung der WSI-Betriebsrätebefragung dennoch sinnvoll, da es sich nach unserer Kenntnis um die einzige Betriebserhebung handelt, bei der neben der Tarifbindung auch der gewerkschaftliche Organisationsgrad der Beschäftigten (sowie auch der Betriebsratsgremien) erhoben wird.

Wie Abbildung 1 belegt, haben wir es bei der arbeitgeberseitigen Erstreckung von Tarifbestimmungen grob gesprochen mit zwei Welten zu tun: erstens die Klein-, Mittelund kleinen Großbetriebe (also insgesamt Betrieben mit weniger als 1.000 Beschäftigten) und zweitens die Schwergewichte mit mehr als 1.000 Beschäftigten. Und während in der „ersten Welt“ die Erstreckungsleistung um die 
ABB. 1

\section{Erstreckung von Verbandstarifverträgen}

Angaben in Prozent*

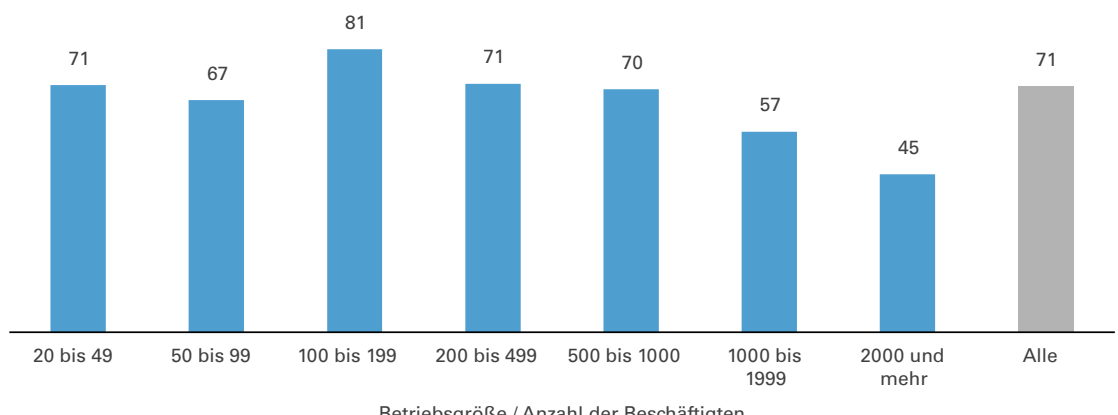

Betriebsgröße / Anzahl der Beschäftigten

*Anteil aller Beschäftigten in der jeweiligen Betriebsgrößenklasse, die auch ohne eigene Gewerkschaftsmitgliedschaft von den Bestimmungen des jeweils geltenden Verbandstarifvertrags profitieren.

Quelle: Berechnung des Autors auf Basis der WSI-Betriebsrätebefragung 2010.

ABB. 2

\section{Gewerkschaftlicher Organisationsgrad der Beschäftigten nach Form der Tarifbindung}

Angaben in Prozent*

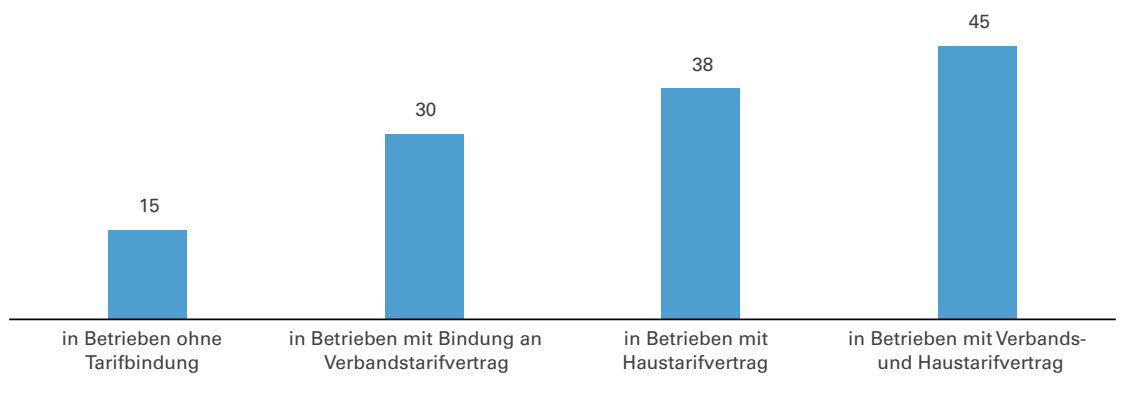

*gerundet.

Quelle: Berechnung des Autors auf Basis der WSI-Betriebsrätebefragung 2010.

$70 \%$ schwankt, sinkt die Bedeutung der freiwilligen Anwendung von Tarifstandards in den beiden Großkategorien auf 57 bzw. 45 \% der Beschäftigten, die auch ohne eigene Gewerkschaftsmitgliedschaft von der Verbandsmitgliedschaft ihres Arbeitgebers profitieren. ${ }^{1}$

Abbildung 2 zeigt, dass gewerkschaftliche Stärke unterschiedlich deutlich mit verschiedenen Formen der Tarifbindung korreliert. Zunächst kann nicht erstaunen, dass mit 14,7\% der gewerkschaftliche Organisationsgrad der Beschäftigten (Mittelwert der betrieblichen Einzelorganisationsgrade) in der Gruppe der Betriebe ohne jede Tarifbindung besonders gering ist. Letztlich handelt es sich hier um das negative Pendant zur Erstreckungsthematik, also um Fälle der für die Tarifpolitik „folgenlosen“ Gewerk- schaftsmitgliedschaft, die am Ende zu keinerlei Tarifbindung führt. Mit 30,4 \% erweist sich hingegen der Beschäftigtenorganisationsgrad in der Gruppe der an einen Verbandstarifvertrag gebundenen Betriebe als vergleichsweise hoch. Besonders auffällig ist darüber hinaus der nochmals höhere Organisationsgrad in den Reihen der Betriebe mit Haustarifvertrag. Dieser beträgt bei Betrieben mit ausschließlicher Bindung an einen Haustarifvertrag 37,8 \% und bei Betrieben, in denen ein Haustarifvertrag (z. B. in Form eines Ergänzungstarifvertrages) zusätzlich zum Verbandstarif gilt, sogar $44,8 \%$. Gerade bei den zuletzt genannten Formen der Bindung an einen Haustarifvertrag wird deutlich, dass es der autonomen gewerkschaftlichen Stärke bedarf, um verbandsabstinente Unternehmen, die sich keinem Verbandstarifvertrag unterwerfen wollten, in die Tarifbindung zu bringen. Der Höchstwert von fast $45 \%$ Organisationsgrad für jene (immerhin 135) Fälle in unserer Analyse, die neben einen Verbandstarif zusätzlich einen Haustarifvertrag anwenden, erklärt sich vermutlich über neue Formen der Gewerkschaftsstrategie. Gewerkschaften wie die IG Metall, aber auch die Gewerkschaft Nahrung, Genuss, Gaststätten (NGG), sind als Teil einer Mobilisierungsstrategie dazu übergegangen, als Voraussetzung für ihre Bereitschaft, vom Verbandstarifvertrag abweichende Regelungen zu verhandeln, die Erzielung eines Mindestorganisationsgrades im Betrieb/Unternehmen zu verlangen.

\section{Innere Erosion durch Mitglied- schaften ohne Tarifbindung?}

War äußere Erosion, verstanden als Resultat der zurückgehenden Mitgliedschaft in Arbeitgeberverbänden, noch das Hauptthema der 1990er Jahre, so traten spätestens nach der Jahrtausendwende mit der inneren Erosion weitere problemverschärfende Prozesse hinzu. Treibende Kraft dieser inneren Erosion war die systematische Entkoppelung zweier Grundprinzipien deutscher Arbeitgeberverbände: der Verbandsmitgliedschaft auf der einen und der Bindung an einen vom Verband geschlossenen Verbandstarifvertrag auf der anderen Seite. Mit der Einführung von sogenannten Mitgliedschaften ohne Tarifbindung (OT) erlaubten nun ausgewählte Verbände ihren Mitgliedern, an der Mitgliedschaft im Verband festzuhalten und weiterhin alle weiteren verbandlichen Dienstleistungen in Anspruch zu nehmen, jedoch gleichzeitig die Bindung an einen geschlossenen Verbandstarifvertrag abzustreifen.

1 Die Berechnungen erfolgten auf Basis der Gesamtzahl der Beschäftigten innerhalb der jeweiligen Größenkategorien und nicht durch Bildung der Mittelwerte der einzelnen betrieblichen Erstreckungsgrade. 
Galten bis dahin Mitgliedschaft und Tarifbindung als zwingend miteinander verknüpfte Grundprinzipien von Arbeitgeberverbänden, so kam es mit der Einführung von OT zur Umprogrammierung von Arbeitgeberverbänden. Tarifpolitik ist in diesen Verbänden nicht mehr das konstitutive Element schlechthin, sondern eine Funktion auf Abruf. Ob bestimmte Tarifstandards für das einzelne Mitglied Geltung erlangen, wird nicht mehr durch die Solidar(oder auch Leidens-)gemeinschaft Arbeitgeberverband als Ganzes entschieden, sondern im Zweifel durch jedes einzelne Mitglied selbst.

Zwar lassen sich OT-ähnliche Bestimmungen bereits in älteren Satzungen finden, wie z. B. jener eines norddeutschen Industrieverbandes aus dem Jahre 1965 (Behrens 2011, S. 138), zur wirklichen Massenbewegung wurde OT allerdings erst in der zweiten Hälfte der 1990er Jahre. Als einer der Pioniere von OT in jüngerer Zeit erwies sich der Verband der holz- und kunststoffverarbeitenden Industrie Rheinland-Pfalz (Völkl 2002, S. 17), welcher bereits im Jahre 1990 eine OT-Mitgliedschaft in der Satzung verankerte und in der Folge sehr offensiv für OT warb (Koch 1999). Schätzungen gehen davon aus, dass heute, mehr als 20 Jahre nach der erstmaligen Einführung von OT-Mitgliedschaften durch deutsche Arbeitgeberverbände, gut die Hälfte aller deutschen Verbände ihren Mitgliedern die Abwahl der Tarifbindung ermöglicht (Behrens 2011, S. 151; Behrens/ Helfen 2010). Hierbei hat sich die Mehrheit dieser Verbände dazu entschiedenen, OT im sogenannten Stufenmodell anzubieten, welches eine Differenzierung verschiedener Mitgliedschaftsformen innerhalb einer einheitlichen Verbandsstruktur vorsieht. Nicht tarifgebundene (OT-)Mitglieder gehören weiterhin dem Verband an, werden aber von der Tarifpolitik ausgenommen. Sie können nach wie vor von der arbeitsrechtlichen Beratung und Prozessvertretung des Verbandes profitieren, nicht jedoch Mitglied einer Tarifkommission werden, sich anderweitig an Abstimmungen zu tariflichen Fragen beteiligen oder gar - sofern vorhanden - Leistungen aus der Streikkasse („Gefahrengemeinschaft") beziehen. Eine kleinere Gruppe von Verbänden, insbesondere die Mitgliedsverbände von Gesamtmetall, betreiben OT im Parallelverbandsmodell (Haipeter/Schilling 2006, S. 54). Hierbei werden tarifgebundene und nicht tarifgebundene Mitglieder in jeweils getrennten Verbänden organisiert.

Für die Frage nach der Bedeutung von OT für die Stellung der Arbeitgeberverbände im deutschen System der industriellen Beziehungen, oder genauer, der möglichen Dualisierung oder Segmentierung ihrer Verankerung, ist es entscheidend, mit welcher strategischen Zielsetzung diese Umprogrammierung der Verbände in Verbindung gebracht wird. Letztlich lassen sich vier Strategiekonzepte unterscheiden, die allerdings zum Teil deutliche Schnittmengen untereinander aufweisen.

$\mathrm{Zu}$ nennen ist hier erstens die Sicht von OT als einer Organisationssicherungsstrategie (Völkl 2002, S. 88; Haipeter 2010, S. 212), welche letztlich als Reaktion auf vorangegan- gene Tendenzen der externen Erosion zu sehen ist. Bevor Mitglieder dem Arbeitgeberverband gänzlich den Rücken zukehren, sollte mit der Einführung von OT-Mitgliedschaften zumindest die Organisationsentwicklung stabilisiert werden (Zimmer 2002, S. 150; Behrens 2011, S. 153). Dieser Perspektive liegt letztlich die Auffassung zugrunde, dass die eigentliche Ursache für die Verbandsabstinenz bei der verbandlichen Tarifpolitik zu suchen ist und nicht etwa in zu hohen Mitgliedsbeiträgen oder unzureichenden anderen Verbandsdienstleistungen. Tarifvertragliche Regelungen seien zu unflexibel und/oder vereinbarte Vergütungen schlichtweg zu hoch (Völkl 2002, S.77f.; Schnabel 2005, S.14f.). Durch die Möglichkeit, Verbandstarifverträge abzuwählen, könnte demnach die Anzahl der Verbandsaustritte verringert werden bzw. jene neuen Mitglieder für den Verband gewonnen werden, die bislang nur deshalb nicht beigetreten waren, weil sie die geltenden Tarifverträge für eher geschäftsschädigend hielten.

In der Tat scheint für einige Arbeitgeberverbände, die sich für die Einführung von OT-Mitgliedschaften entschieden haben, die Rechnung im Sinne der Organisationssicherung aufzugehen. So konnte beispielsweise Gesamtmetall im Zuge der Einführung von OT-Verbänden durch seine Landes- und Regionalverbände einen leichten Mitgliederzuwachs verbuchen. Während es im Bereich der tarifgebundenen Mitglieder weiterhin zu kontinuierlichen Mitgliederverlusten kam, konnte diese Entwicklung durch die OT-Verbände mehr als kompensiert werden. Organisierte Gesamtmetall im Jahre 2005 noch 5.861 Unternehmen in den Reihen seiner Mitgliedsverbände, so war die Mitgliederzahl zum Ende des Jahres 2011 auf 6.565 angestiegen. Im gleichen Zeitraum allerdings sank die Zahl der tarifgebundenen Mitgliedsunternehmen von 4.429 auf 3.652 (Angaben laut Gesamtmetall).

Zweitens kann OT aber auch als eine Art Legalisierungsstrategie verstanden werden, bei der die Einführung von OT-Mitgliedschaften als Versuch gesehen werden kann, vom Tarifvertrag abweichende (rechtswidrige) Regelungen zu legalisieren (entsprechende Hinweise finden sich bei Rosdücher 1997, S. 174). Die Einführung der tarifbindungsfreien Mitgliedschaft im Verband ist in dieser Argumentation als ein Angebot an jene Mitgliedsunternehmen zu verstehen, die sich vor der Einführung dieser Alternative gezwungen sahen, Tarifbestimmungen (z. B. durch abweichende Vereinbarungen mit den Betriebsräten) zu unterlaufen.

Drittens lässt sich OT als tarifpolitisches Druckmittel interpretieren. Hierbei könnte allein schon die glaubhaft vorgetragene Drohung des Wechsels von Mitgliedern in die OT-Mitgliedschaft ein wertvolles Druckmittel in den Händen der Arbeitgeberverbände darstellen, welches im Kontext von Tarifauseinandersetzungen die Konzessionsbereitschaft der Gewerkschaften maßgeblich erhöhen könnte (Haipeter/ Schilling 2006, S. 61; Bispinck 2006; Schroeder/Silvia 2003, S. 264). So kann innerhalb der Dramaturgie einer Tarifbewegung bereits der glaubhafte Hinweis, dass sich ein 
beachtlicher Teil der Mitglieder gezwungen sehen könnte, quasi über Nacht dem Geltungsbereich des Tarifvertrages $\mathrm{zu}$ entfliehen, wenn der Tarifgegner weiterhin auf unangemessenen Forderungen beharren würde, durchaus Wirkung zeigen. Allerdings: So wirksam diese Drohkulisse im Einzelnen auch sein mag, so ist aber auch davon auszugehen, dass dieses Schwert mit der Häufigkeit seiner Nutzung immer stumpfer wird. Wie man es auch wendet, das Drohpotenzial nutzt sich ab: Folgt der Drohung mit dem Wechsel in OT kein entsprechendes Verhalten der Mitglieder, wird die Drohung zunehmend unglaubwürdig. Machen die Arbeitgeberverbandsmitglieder hingegen mit ihrer Drohung Ernst und wechseln in OT, verringert sich das Potenzial möglicher Wechselkandidaten in den folgenden Tarifrunden.

Dies leitet über zur vierten und letzten strategischen Motivation von tarifbindungsfreien Mitgliedschaften, der Exit-Strategie. Bei der Exit-Strategie ist die Bereitstellung der Möglichkeit, die Tarifbindung abzustreifen, letztlich als Einstieg in den Ausstieg aus dem Verbandstarifvertrag zu werten. So findet sich beispielsweise in der Studie von Völkl (2002, S. 107) der Verband der Pfälzischen Sägewerke, der sich entschlossen hat, keine Tarifverhandlungen durchzuführen und stattdessen lediglich tarifpolitische Verbandsempfehlungen ausspricht. Ein solcher Ausstieg ist auch als gradueller Prozess denkbar, bei dem der Anteil der OTMitglieder im Verband so weit ansteigt, dass letztlich die verbleibende Minderheit der tarifgebundenen Mitglieder kaum mehr in der Lage ist, einheitliche Standards innerhalb der Branche durchzusetzen. Eine solche Aushöhlung wäre sicherlich nicht konfliktfrei, würde aber die Verbandsführung aus der Schusslinie nehmen. Die Entscheidung über den tarifpolitischen Systemwechsel würde in die Hände der einzelnen Mitglieder selbst gelegt, die durch ihre kumulierten Einzelentscheidungen Fakten schaffen.

Die Folgen dieser unterschiedlichen Strategiebündel für die Frage, wie sich die Einführung von OT-Mitgliedschaften auf die Verfassung deutscher Arbeitgeberverbände auswirkt, könnten unterschiedlicher kaum sein. Während eine ExitStrategie bewusst einen Pfadbruch heraufbeschwört, indem sie einen zentralen Stützpfeiler des deutschen Systems der Verbandstarifverträge einzureißen sucht, bewegen sich die Organisationssicherungsstrategie sowie die Nutzung von OT als tarifpolitisches Druckmittel noch deutlich innerhalb der Logik des bestehenden Systems. Die Exit Strategie - sofern sie Erfolg hat - wird letztlich Arbeitgeberverbände überflüssig machen, aus ihnen werden reine sozialpolitische Lobby- und Serviceorganisationen. Der Versuch, mit Rückgriff auf eine reine Organisationssicherungsstrategie OT zur Stabilisierung der Mitgliedschaft nutzen zu wollen, kann aber durchaus dahin gehend interpretiert werden, dass ein Kern, bestehend aus tarifwilligen Mitgliedsunternehmen in ihrer Mehrzahl große und mittelgroße Unternehmen stabilisiert werden soll. Dies erfolgt, indem die Beitragseinnahmen stabil gehalten und OT-Mitglieder immerhin recht nahe am Verbandsgeschehen und somit auch der generellen
Verbandspolitik gehalten werden. Dass diese mit den jeweiligen Strategien assoziierten Folgen letztlich auch eintreten, ist damit noch nicht ausgemacht, denn gerade die langfristigen Effekte der Einführung von OT-Mitgliedschaften sind nur schwer zu bestimmen. Auf diesen Aspekt wird im Folgenden zurückzukommen sein.

\section{Zukunft von Arbeitgebermacht?}

Wie in den vorangegangenen Abschnitten gezeigt wurde, vollzieht sich die Entwicklung deutscher Arbeitgeberverbände innerhalb des Spannungsverhältnisses zweier Entwicklungslinien: der äußeren Erosion durch den beharrlichen und anhaltenden Rückgang von Tarifdeckungsraten sowie der inneren Erosion durch die sukzessive Umprogrammierung eines beachtlichen Teils der deutschen Arbeitgeberverbände. Beide Prozesse sind miteinander verknüpft, können aber nicht einfach aus dem jeweils anderen abgeleitet werden. So ist die Einführung von OT-Mitgliedschaften bei einem Teil der betroffenen Verbände als ein Versuch zu sehen, den schleichenden Verfall der Mitgliedschaftsbasis anzuhalten, wenn nicht sogar rückgängig zu machen (mit bislang offenem Ausgang), jedoch greifen die Konsequenzen dieser Versuche weit über Fragen der Mitgliedschaftsentwicklung hinaus, indem die grundlegenden Funktionen und Ressourcen der Verbände verändert werden.

Die entscheidende Frage ist nun, wie beide Dimensionen der Erosion zusammenwirken und welche die prognostizierbaren Entwicklungslinien sein werden. Erste Anhaltspunkte hierzu ergeben sich aus den Daten der Befragung „Wirtschaftsverbände in Deutschland 2006“, die an der RWTH Aachen durchgeführt wurde (zu den Ergebnissen siehe Behrens/Helfen 2010). Wie Tabelle 1 zeigt, werden Verbände ohne die Möglichkeit der Abwahl der Bindung an den Verbandstarifvertrag mit zwei unterschiedlichen Formen von OT verglichen. Die eher „defensive“ Form von OT ohne Sonderkündigungsrecht zwang zum Zeitpunkt der Datenerhebung solche Unternehmen, die die Tarifbindung abstreifen wollten, dazu, die reguläre satzungsmäßige Kündigungsfrist (zumeist drei oder sechs Monate) einzuhalten. Da somit zwischen der Erklärung des Übertritts in die neue Mitgliedschaftsform und deren Wirksamwerden ein längerer Zeitraum liegt, ist OT in dieser Variante kaum mehr als unmittelbares Druckmittel während der Tarifrunden zu gebrauchen. Bei der zweiten Variante, OT in Kombination mit einem Sonderkündigungsrecht, verhält sich dies anders. Mit dem Sonderkündigungsrecht wird die Kündigungsfrist beim Übertritt von Mitgliedern in die OTMitgliedschaft deutlich verkürzt, in den meisten Fällen entfällt sie vollständig. Da hiermit die Abwahl der Verbandstarifbindung sofort wirksam werden kann (was selbstverständlich die Nachbindung bzw. Nachwirkung der geltenden 
Tarifverträge nicht aushebelt), ist in diesem Modell der Druck auf den jeweiligen Tarifpartner erheblich.

Wie aus Tabelle 1 hervorgeht, ergibt sich bezüglich des Zusammenhangs zwischen der Einführung von OT-Mitgliedschaften und der relativen Stärke der jeweiligen Arbeitgeberverbände kein eindeutiger Zusammenhang. In der defensiven Variante von OT ist zwar der Mitgliederorganisationsgrad der Verbände etwas höher als in der Referenzgruppe ohne jede OT-Regelung in der Satzung, in der radikalen OT-Variante allerdings verhält es sich genau umgekehrt: Hier liegt der durchschnittliche Organisationsgrad mit $53 \%$ immerhin sechs Prozentpunkte unter dem Wert der Verbände ohne OT.

Jedoch sagt der Organisationsgrad als Maß für den Anteil der Verbandsmitglieder an den möglichen Mitgliedsunternehmen innerhalb einer Verbandsdomäne noch nichts darüber aus, wie sehr Verbände ihre gegenwärtige Mitgliederentwicklung als Problem empfinden müssen. In den drei letzten Zeilen der Tabelle befinden sich daher für die drei Gruppen von Verbänden auch Angaben darüber, wie sich die Mitgliedschaft im Zeitraum von 2001 bis 2005 entwickelt hat. Hier zeigen sich zunächst recht deutliche Unterschiede zwischen Verbänden ohne OT-Option auf der einen Seite und beiden Varianten von OT auf der anderen. Berichteten die befragten Geschäftsführungen in Verbänden ohne OT in $45 \%$ der Fälle von zurückgehender Mitgliedschaft, so betrug dieser Anteil in den beiden OT-Gruppen $64 \%$ (defensive OT-Variante) bzw. 83 \% (offensive OT-Variante mit Sonderkündigungsrecht). Die Angaben zu wachsender Verbandsmitgliedschaft verhalten sich spiegelbildlich: $26 \%$ aller Verbände ohne OT verzeichneten in den fünf Jahren vor der Befragung eine Zunahme ihrer Mitgliedschaft, in der Gruppe der Verbände mit defensiver OT-Variante waren dies $14 \%$, bei offensiver OT-Variante $6 \%$. Nun muss allerdings auf eine mögliche Unschärfe der präsentierten Daten hingewiesen werden. Da für eine Mehrzahl der untersuchten Verbände keine Informationen darüber vorliegen, zu welchem genauen Zeitpunkt OT-Mitgliedschaften eingeführt wurden, können die Daten nur mit großer Vorsicht interpretiert werden. So wäre es beispielsweise auch vorstellbar, dass zurückgehende Mitgliederzahlen eine Folge der Einführung von OT sind. Für die gegenteilige Annahme, dass OT letztlich auch eine mögliche (zudem erfolgreiche) Antwort auf zurückgehende Mitgliederzahlen ist, sprechen letztlich auch die im vorangegangenen Abschnitt präsentierten Erfahrungen im Bereich der Mitgliedsverbände von Gesamtmetall.

Somit lassen sich die Daten wohl dahin gehend interpretieren, dass OT für eine Mehrzahl der Verbände in Verbindung mit vorangegangenen Mitgliederverlusten zu sehen ist. Insofern erfährt hier die Sicht von OT als eine Art Organisationssicherungsstrategie Unterstützung. Und wie die Daten weiterhin andeuten, neigen Verbände mit Intensivierung des von Mitgliederverlusten ausgehenden Drucks auch eher dazu, eine besonders radikale Spielart von OT zur Anwendung zu bringen und den Übertritt in die tariflose Ver-

\section{TABELLE 1}

OT und Mitgliederentwicklung der Arbeitgeberverbände 2001 - 2005

Angaben in Prozent*

\begin{tabular}{l|c|c|c}
\hline & Ohne OT & $\begin{array}{c}\text { OT ohne Sonder- } \\
\text { kündigungsrecht }\end{array}$ & $\begin{array}{c}\text { OT mit Sonder- } \\
\text { kündigungsrecht }\end{array}$ \\
\hline Mitglieder Organisationsgrad & 59 & 62 & 53 \\
\hline
\end{tabular}

\begin{tabular}{l|c|c|c}
\hline Mitgliederentwicklung: sinkend & 45 & 64 & 83 \\
\hline Mitgliederentwicklung: stagnierend & 29 & 23 & 11 \\
\hline Mitgliederentwicklung: wachsend & 26 & 14 & 6 \\
\hline
\end{tabular}

* Rundungsbedingt addieren sich nicht alle Prozentanteile in den Spalten zu $100 \%$ auf.

Quelle: Wirtschaftsverbände in Deutschland (2006), zitiert nach Behrens/Helfen (2010).

bandsmitgliedschaft ohne lästige Restriktionen zu versehen. „Organisationssicherung" gestaltet sich in dieser Gruppe wohl eher als Prozess des „rette sich wer kann“. Es kann im Falle der radikalen OT-Variante nur vermutet werden, dass Verbände hier tarifmüden Mitgliedern keine lange Warteperiode zumuten wollen. Der von der schlechten Mitgliederentwicklung ausgehende Handlungsdruck ist so groß, dass man nach einer schnell wirksamen Medizin gesucht hat.

Die eingangs aufgeworfene Frage des Zusammenwirkens beider Prozesse der inneren und äußeren Erosion beantwortet sich allerdings nicht allein auf der Ebene des politischen Handelns der Verbände, sondern darüber hinaus auch über die Entscheidungen der einzelnen Unternehmen, Mitglied im Verband zu sein (oder nicht) bzw. als Verbandsmitglied in die OT-Mitgliedschaft zu wechseln (oder auch nicht). Im Resultat zeigen sich hier gegenseitig verstärkende Prozesse: So wissen wir seit Langem, dass die Wahrscheinlichkeit, dass ein Unternehmen einem Arbeitgeberverband angehört, mit seiner Größe zunimmt. Gleichzeitig ist auch bekannt, dass - insofern durch den Verband überhaupt angeboten - OT-Mitgliedschaften bevorzugt von kleinen und mittelgroßen Unternehmen in Anspruch genommen werden. Nach Angaben von Gesamtmetall nahmen im Jahr $201042 \%$ aller Mitgliedsunternehmen der angeschlossenen Landes- und Regionalverbände eine OTMitgliedschaft in Anspruch, durch die Unternehmen wurden allerdings nur $17 \%$ aller Beschäftigten der Mitgliedsunternehmen erfasst (Angaben nach Gesamtmetall). Auf den Punkt gebracht bedeutet diese zweistufige Entwicklung, dass kleine und mittelgroße Unternehmen eher den Arbeitgeberverbänden fernbleiben, sollten sie aber den Verbänden dennoch angehören, weisen sie eine größere Wahrscheinlichkeit auf, sich der dadurch begründeten Tarifbindung zu entziehen. Eine bereits vorher bestehende Dualisierung der Unternehmenslandschaft in Großunternehmen auf der einen Seite, für die die Kerninstitutionen der Arbeitsbeziehungen Gültigkeit haben, und auf der anderen Seite 
kleine und mittelgroße Einheiten, die sich diesen entziehen, dürfte sich also auf diesem Wege weiter etablieren. Dieser Prozess wird auch, wie im zweiten Abschnitt gezeigt, durch die Tatsache verstärkt, dass Gewerkschaften in den meisten klein- und mittelbetrieblichen Strukturen bislang kaum eigene Organisationsmacht aufbringen können. Der von den Gewerkschaften ausgehende stabilisierende Einfluss auf das Gesamtsystem entfällt hier.

\section{Fazit}

Wie die vorangegangene Analyse belegt, spricht vieles für die in Teilen der „Dualisierung/Segmentierung-Literatur“ vertretene These, dass der Schutz sozialer Rechte der Beschäftigten, wie er z. B. durch die Institution der Verbandstarifverträge gewährt wird, zunehmend brüchig wird und längst nicht mehr für alle Beschäftigtengruppen gilt. Zumindest auf der Ebene ihrer zentralen Beobachtung, wonach es auch in Deutschland (ebenso wie in anderen europäischen Ländern) zur Herausbildung von koexistierenden, gleichwohl sehr unterschiedlichen Welten der Arbeitsbeziehungen kommt, ist dem Ansatz zuzustimmen. Für den zweiten Bestandteil der Dualisierungsthese, wonach sich das Zentrum zulasten der Peripherie stabilisiert, finden sich allerdings kaum Belege. So konnte am Beispiel der inneren und äußeren Erosion der Arbeitgeberverbände gezeigt werden, dass die doch recht schlichte Annahme, hier würde sich ein ökonomisch hoch-potenter Kern auf Kosten einer weitgehend schutzlos ausgelieferten Peripherie stabilisieren, zu kurz greift. Zwar vollzieht sich der Rückzug des Verbandstarifvertrages als einer Kerninstitution der deutschen Arbeitsbeziehungen recht deutlich entlang einer Bruchlinie Großbetriebe-/unternehmen auf der einen und klein- und mittelbetriebliche Strukturen auf der anderen Seite, ein solcher Prozess kann aber keineswegs vorwiegend als Produkt des strategischen Handelns von Arbeit und Kapital auf der betrieblichen Ebene verstanden werden. Die Einführung von Mitgliedschaften ohne Tarifbindung vollzieht sich als strategische Entscheidung auf Verbandsebene und verstärkt im Resultat die Schwäche der Verbandstarifbindung in den Reihen der Klein- und Mittelbetriebe. Zugespitzt könnte man hier feststellen, dass sich in Fragen der Tarifbindung nicht etwa ein imaginäres Zentrum zulasten der Peripherie stabilisiert; vielmehr öffnet eine Vielzahl der Verbände alle Schleusen, um es der Peripherie einfacher zu machen, sich dem Wettbewerbsdruck auf dem Wege der Aufgabe einheitlicher Tarifbedingungen zu entziehen. Wie die geschilderten Befunde quantitativer Forschung zur Tarifbindung weiterhin belegen, wirken sich strukturelle Einflüsse der Unternehmen sehr deutlich auf die Fähigkeit der Arbeitgeberverbände aus, ihre Mitgliederbasis zu erneuern und schon immer bestehende Effekte der regulären Mitgliederfluktuation auszugleichen. Insofern spricht vieles dafür, dass deutsche Arbeitgeberverbände einen Teil ihrer Fähigkeit eingebüßt haben, ihre Mitgliedschaft kontinuierlich zu erneuern. Für die in Teilen der Dualisierungsliteratur zu findende These, eine unheilige Allianz aus Kapital und Arbeit der Großunternehmen des verarbeitenden Gewerbes würde sich auf Kosten „der anderen“ stabilisieren, finden sich keine Belege. Wie die hier präsentierten Daten zur Erstreckungsleistung deutscher Arbeitgeberverbände andeuten, fehlt es den Gewerkschaften in weiten Teilen der tariflich ungebundenen Betriebe und Unternehmen schlichtweg an Mitgliederstärke, um Arbeitgeber in die Tarifbindung zu zwingen. Die deutlich unterschiedlichen gewerkschaftlichen Organisationsgrade in den Reihen der tarifgebundenen Mitgliedsunternehmen belegen weiterhin, dass der eigenständigen strategischen Orientierung in Fragen der Bindung an einen Verbandstarif eine große Bedeutung zukommt. Wenn Arbeitgeber dies mehrheitlich wollen, kommt auch dann ein Verbandstarifvertrag zur Anwendung, wenn sie seitens der Gewerkschaft wenig Gegenwehr zu befürchten haben. Wie sich hier zeigt, wird die Auseinandersetzung um die deutschen Kerninstitutionen der Arbeitsbeziehungen nicht vorwiegend auf der Ebene des Betriebs oder Unternehmens und der dort bestehenden Koalitionen geführt, sondern in besonderem Maße auf der Ebene des Verbandes.

\section{LITERATUR}

Behrens, M. (2011): Das Paradox der Arbeitgeberverbände. Von der Schwierigkeit, durchsetzungsstarke Unternehmensinteressen kollektiv zu vertreten, Berlin

Behrens, M./Helfen, M. (2009): Innerverbandliche Heterogenität und die Vertretungswirksamkeit deutscher Arbeitgeberverbände, in: Industrielle Beziehungen 16 (1), S. 5-24

Behrens, M./Helfen, M. (2010): Employers' Exit from Multi-Employer Bargaining. Organizational Change in German Employers' Associations, in: Proceedings of the 9th IIRA European Congress, 29 June - 1 July 2010, Copenhagen Behrens, M./Helfen, M. (2012): Variety in Social Partnership in Corporatist Germany: A look at employers' associations' attitudes. Presentation at the 16th World Congress of the International Labor and Employment Relations Association, Philadelphia, http://ilera2012.wharton.upenn.edu/RefereedPapers/ HelfenMarkus\%20MartinBehrens.pdf

Bispinck, R. (2006): Abschied vom Flächentarifvertrag? Der Umbruch in der deutschen Tariflandschaft, in: WSI (Hrsg.): WSI-Tarifhandbuch 2006, Frankfurt a. M., S. 39-66

Bispinck, R. (2012): Allgemeinverbindlicherklärung von Tarifverträgen - vom Niedergang zur Reform?, in: WSI-Mitteilungen 65 (7), S. 496-507, http://www. boeckler.de/wsimit_2012_07_bispinck.pdf

Ellguth, P./Kohaut, S. (2010): Auf der Flucht? Tarifaustritte und die Rolle von Öffnungsklauseln, in: Industrielle Beziehungen 17 (4), S. 345-371

Ellguth, P./Kohaut, S. (2012): Tarifbindung und betriebliche Interessenvertretung. Aktuelle Ergebnisse aus dem IAB-Betriebspanel 2011, in: WSI-Mitteilungen 65 (4), S. 297-305, http://www.boeckler.de/wsimit_2012_04_ellguth.pdf Haipeter, Th. (2010): OT-Mitgliedschaften und OT-Verbände, in: Schroeder, W./ Weßels, B. (Hrsg.): Handbuch Arbeitgeber- und Wirtschaftsverbände in Deutschland, Wiesbaden, S. 209-219

Haipeter, Th./Schilling, G. (2006): Arbeitgeberverbände der Metall- und Elektroindustrie. Tarifbindung, Organisationsentwicklung und Strategiebildung, Hamburg

Hassel, A. (2011): The paradox of liberalization - Understanding dualism and the recovery of the German political economy: LSE 'Europe in Question', Discussion Paper Series 42/2011, London

Koch, G. (1999): Flucht in die OT-Mitgliedschaft, in: Personalwirtschaft 9/1999, S. $47-52$

Lehmann, K. (2002): Stabilität und Veränderung der Flächentarifbindung von Arbeitgebern in Deutschland, Münster

Palier, B./Thelen, K. (2010): Institutionalizing Dualism: Complentaries and Change in France and Germany, in: Politics \& Society 38 (1), S. 119-148 Rosdücher, J. (1997): Arbeitsplatzsicherheit durch Tarifvertrag. Strategien, Konzepte, Vereinbarungen, München/Mehring

Schnabel, C. (2005): Gewerkschaften und Arbeitgeberverbände: Organisationsgrade, Tarifbindung und Einflüsse auf Löhne und Beschäftigung: FriedrichAlexander-Universität Erlangen-Nürnberg, Lehrstuhl für VWL, Diskussionspapier (34), Nürnberg

Schnabel, C./Wagner, J. (1996): Ausmaß und Bestimmungsgründe der Mitgliedschaft in Arbeitgeberverbänden: Eine empirische Untersuchung mit Firmendaten, in: Industrielle Beziehungen 3 (4), S. 293-306

Schroeder, W./Ruppert B. (1996): Austritte aus Arbeitgeberverbänden. Eine Gefahr für das deutsche Modell?, Marburg

Schroeder, W./Silvia, S. (2003): Gewerkschaften und Arbeitgeberverbände, in: Schroeder, W./Weßels, B. (Hrsg.): Die Gewerkschaften in Politik und Gesell- 
schaft der Bundesrepublik Deutschland. Ein Handbuch, Wiesbaden,

S. $244-270$

Thelen, K. (2000): Why German Employers Cannot Bring Themselves to Dismantle the German Model, in: Iversen, T./Pontusson, J./Soskice, D. (Hrsg.): Union, Employers, and Central Banks. Macroeconomic Coordination and Institutional Change in Social Market Economies, Cambridge, S. 138-169

Völkl, M. (2002): Der Mittelstand und die Tarifautonomie. Arbeitgeberverbände zwischen Sozialpartnerschaft und Dienstleistungen, München/Mehring Zimmer, S. (2002): Jenseits von Arbeit und Kapital? Unternehmerverbände und Gewerkschaften im Zeitalter der Globalisierung, Opladen
AUTOR

MARTIN BEHRENS, PD Dr., Wissenschaftler im Wirtschafts- und Sozialwissenschaftlichen Institut (WSI) in der Hans-Böckler-Stiftung. Arbeitsschwerpunkte: Vergleichende industrielle Beziehungen, Arbeitgeberverbände, betriebliche Mitbestimmung.

@ martin-behrens@boeckler.de 